\title{
Experimental visualization of aerodynamic sound sources using parallel phase-shifting interferometry
}

\author{
Risako Tanigawa $^{1} \cdot$ Kohei Yatabe $^{1} \cdot$ Yasuhiro Oikawa $^{1}$
}

Received: 15 January 2020 / Revised: 24 July 2020 / Accepted: 19 August 2020 / Published online: 2 September 2020

(c) The Author(s) 2020

\begin{abstract}
Aerodynamic sound is one of the causes of noise in high-speed trains, automobiles, and wind turbines. To investigate the characteristics of aerodynamic sound generation, measurements around the sound sources are required. Aerodynamic sound is typically measured using microphones. However, microphones cannot capture the near-field of aerodynamic sound because they become new noise sources inside the air flow. To observe the aerodynamic sound near-field, we performed two-dimensional visualization of aerodynamic sound using an optical method. The optical method used in this research, parallel phase-shifting interferometry (PPSI), can detect the pressure within the measurement area as variations of the phase of light. PPSI can therefore visualize the pressure fields. We visualized both the sound pressure and flow components of the sound generated by flow around a square cylinder and flat plates. The visualized pressure fields are provided as animations in the online resources. Analysis of the sound and flow component time variations confirmed the correlations between them.
\end{abstract}

\section{Graphic abstract}
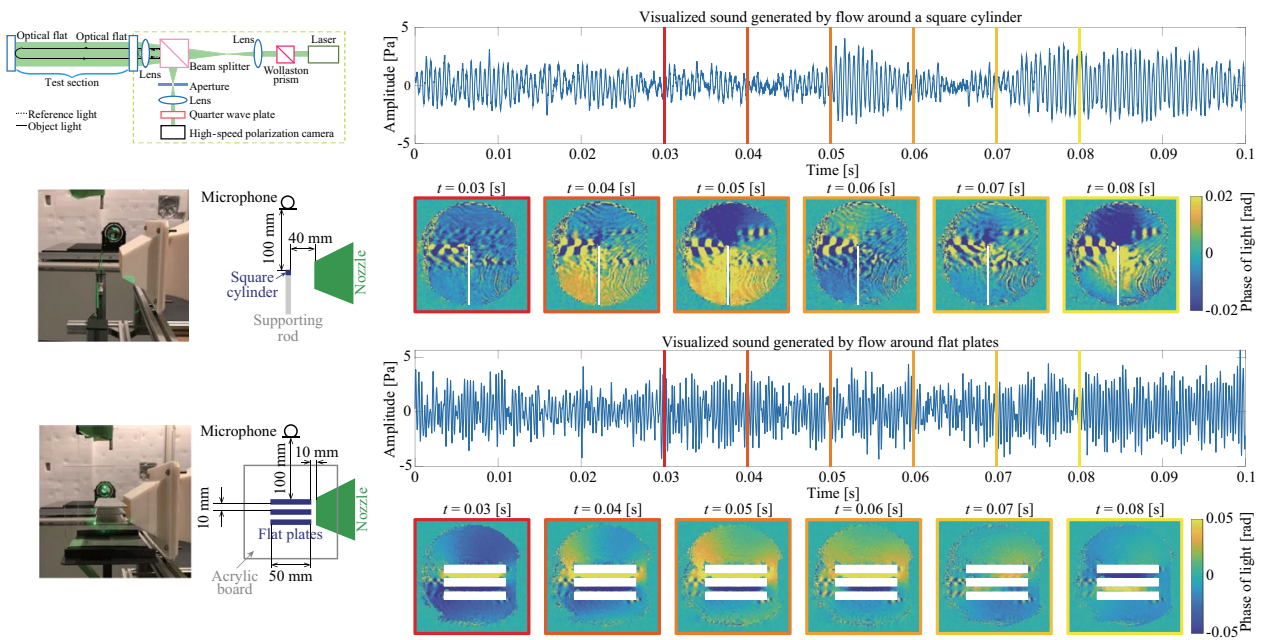

Electronic supplementary material The online version of this article (https://doi.org/10.1007/s00348-020-03038-x) contains supplementary material, which is available to authorized users.

Risako Tanigawa

risako@fuji.waseda.jp

Extended author information available on the last page of the article

\section{Introduction}

Aerodynamic sound is one of the causes of noise in highspeed trains, automobiles, and wind turbines. To reduce such undesired noise, it is important to understand the generation process of the aerodynamic sound. Microphones and microphone arrays are commonly used for measuring radiated aerodynamic sounds. However, they cannot capture sounds inside air flows because microphones installed 
inside the air flow become new noise sources. Windscreens and nose cones are commonly used to handle this difficulty (van den Berg 2006). Although they can reduce the influence of wind noise, their existence induces measurement errors. Hence, non-intrusive measurements are required to measure the near-field of aerodynamic sound without disturbing the flow fields.

Non-intrusive flow visualization for measuring flow fields has been performed using optical methods such as particle image velocimetry (PIV) (Adrian 1991) and particle tracking velocimetry (PTV) (Adamczyk and Rimai 1988). These visualization methods can estimate the causes of the aerodynamic sound generation from the flow velocity. However, they cannot directly visualize sound because sound consists of pressure fluctuations. Interferometric techniques have been applied to measure the sound pressure non-intrusively (Løkberg 1994; Oikawa et al. 2005; Torras-Rosell et al. 2012). These techniques can capture the pressure field by measuring the differences in the optical path lengths caused by variations in the refractive index, which is related to the medium density. These methods are effective for sound fields that are difficult to measure, such as around sound sources, narrow spaces, high-temperature fields, and inside flow fields owing to their non-contact nature (Hargather et al. 2010; Ishikawa et al. 2016; Yatabe et al. 2016).

In this study, we visualized aerodynamic sound using parallel phase-shifting interferometry (PPSI) to visualize the near-field of the aerodynamic sound. PPSI is an optical measurement method that can capture pressure fields instantaneously and quantitatively (Ishikawa et al. 2016). We conducted two experiments to visualize the aerodynamic sound radiated from a square cylinder and flat plates. Sound around the sources and the air flow components that are important for sound generation were visualized.

\section{Methods}

\subsection{Optical sound measurement}

Optical sound measurement utilizes the changes in light phase due to the density fluctuations in sound waves. The light phase $\phi$ is determined by the refractive index of air $n$ :

$\phi(\boldsymbol{r}, t)=k \int_{L(\boldsymbol{r})} n(\boldsymbol{l}, t) \mathrm{d} \boldsymbol{l}$,

where $r$ is the position vector, $t$ is the time, $k$ is the light wavenumber, and $L$ is the optical path. The refractive index of air $n$ is related to the sound pressure $p$ by

$n(\boldsymbol{r}, t) \simeq n_{0}+\frac{n_{0}-1}{\gamma p_{0}} p(\boldsymbol{r}, t)$,

where $n_{0}$ is the atmospheric refractive index, $p_{0}$ is the atmospheric pressure, and $\gamma$ is the specific heat ratio of air. From Eqs. (1) and (2), we can determine the relation between the sound pressure $p$ and the light phase influenced by the sound $\phi_{p}$ as

$\phi_{p}(\boldsymbol{r}, t)=k \frac{n_{0}-1}{\gamma p_{0}} \int_{L(\boldsymbol{r})} p(\boldsymbol{l}, t) \mathrm{d} \boldsymbol{l}$.

Hence, the integrated sound pressure along the optical path can be obtained by observing the light phase.

\subsection{Parallel phase-shifting interferometry}

Directly observing the light phase is difficult because the frequency of light is very high. Interferometry is a major method for observing the light phase through light interference. In particular, the PPSI system used in this research (Ishikawa et al. 2016) realizes the parallel phase-shifting method (Millerd et al. 2004; Awatsuji et al. 2004) by combining a high-speed polarization camera (Onuma and Otani 2014) with a polarization interferometer. The parallel phaseshifting method enables us to capture the light phase instantaneously. A schematic of the PPSI system used in this work is shown in Fig. 1. Light is emitted from a continuous-wave
Fig. 1 PPSI system used in this study. The sound fields inside the test section were observed

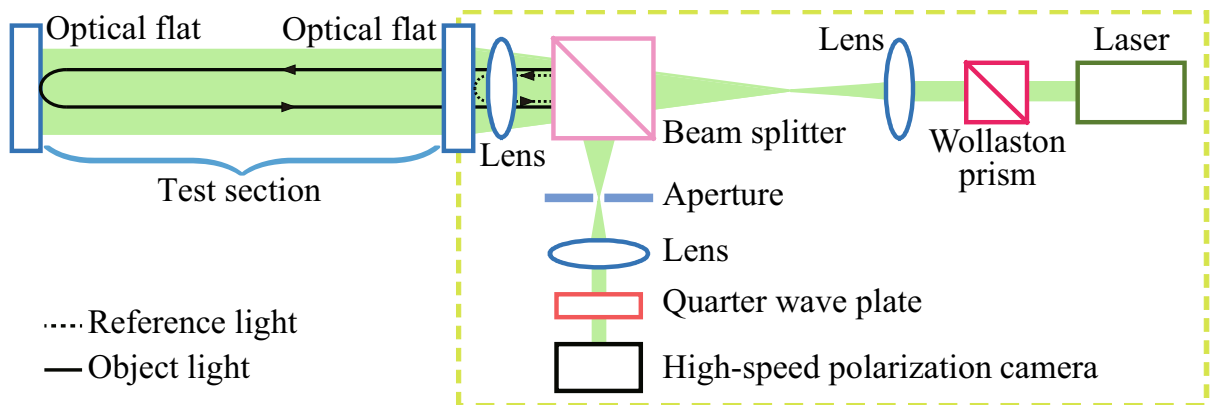


YAG laser with a wavelength of $532 \mathrm{~nm}$. The light is split into two linear polarized light beams by the Wollaston prism. The light beam passing into the test section is expanded to a diameter of $100 \mathrm{~mm}$ and divided by the optical flat in front of the test section into two paths to form the reference and object lights. The reference light is reflected by the optical flat whereas the object light passes into the test section and its phase is changed according to the pressure in the optical path. The object light is combined with the reference light and the phase differences between these two lights are encoded into the polarization state. The combined light is changed into circularly polarized light by the quarterwave plate. The light passes through four linear polarizers with different principal axis orientations associated with each pixel on the image sensor in the high-speed camera (CRYSTA PI-1P, Photron). The maximum measurement area is a $100 \mathrm{~mm}$ diameter circle and the maximum frame rate is $1.5 \mathrm{M}$ frames per second.

\section{Experimental setup}

We conducted two visualization experiments on the aerodynamic sound generated by flow around (a) a square cylinder and (b) flat plates. The aerodynamic sound generated by the flow around a square cylinder is a simple aerodynamic sound. Experimental and numerical investigations (Knisely 1990; Yokoyama and Iida 2017) showed that the sound generated by the flow around a square cylinder is a dipole sound centered on the cylinder. The aerodynamic sound generated by the flow around flat plates resonates between the plates. This resonant sound is difficult to capture with a microphone. Experiments and computations (Yokoyama et al. 2013) for the flat plates showed that synchronized vortex sheddings downstream contribute to the increase in the resonance between the plates. The setups of these experiments are shown in Fig. 2. The nozzle outlet was $100 \mathrm{~mm}$ in width and $30 \mathrm{~mm}$ in height.

The experimental setup for visualizing the sound generated by the flow around a square cylinder is shown in Fig. 2a. The height and width of the square cylinder were $2 \times 2 \mathrm{~mm}$ and the depth was $262 \mathrm{~mm}$. The square cylinder was installed $40 \mathrm{~mm}$ away from the nozzle outlet. In order to fix the position of the square cylinder, supporting rods, which were outside of the flow, were installed at both ends of the cylinder. A 1/4" Free-field Microphone (Type 46BE, G.R.A.S.) with a $1 / 4$ " preamplifier (Type $26 \mathrm{CB}$, G.R.A.S.) was installed $100 \mathrm{~mm}$ above the square cylinder for reference measurements. The frame rate of the high-speed camera was set to 20, 000 frames per second and the measuring time was $0.10 \mathrm{~s}$.

The experimental setup for visualizing the sound generated by the flow around flat plates is shown in Fig. 2b. Three flat plates, each with a width of $50 \mathrm{~mm}$ and thickness of 2 $\mathrm{mm}$, were used. Transparent acrylic boards with holes for the plates were used to fix these plates and visualize the sound between them. The distance between the acrylic boards was $140 \mathrm{~mm}$, and the spacings between the flat plates were 10 $\mathrm{mm}$. The flat plates were installed $10 \mathrm{~mm}$ away from the nozzle outlet. The same type of microphone as the square cylinder experiment was installed $100 \mathrm{~mm}$ above the top of the flat plates for reference measurements. The frame rate of the high-speed camera was set to 20, 000 frames per second and the measuring time was $0.10 \mathrm{~s}$.

The flow velocity profile of the nozzle outlet is shown in Fig. 2c. The flow velocity was measured using a hot-wire probe at the measurement points indicated by the red circles. The flow velocities averaged over $10 \mathrm{~s}$ at each point are plotted. The flow velocity at the center position was $24.3 \mathrm{~m} / \mathrm{s}$. The Reynolds number was $3.12 \times 10^{3}$.

The sound spectra captured by the microphone are shown in Fig. 3. The sound radiated from the flow around
Fig. 2 Experimental setups of sound visualization generated by flow around a a square cylinder and $\mathbf{b}$ flat plates, $\mathbf{c}$ shows the flow velocity of the nozzle outlet used in this study (a)

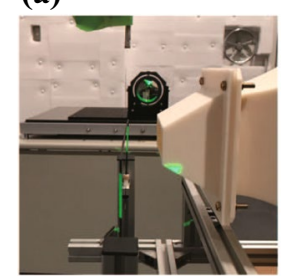

(b)

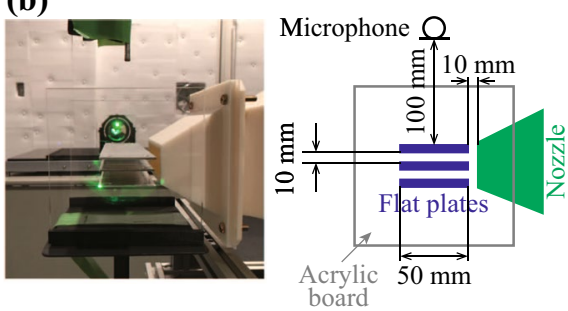

(c)
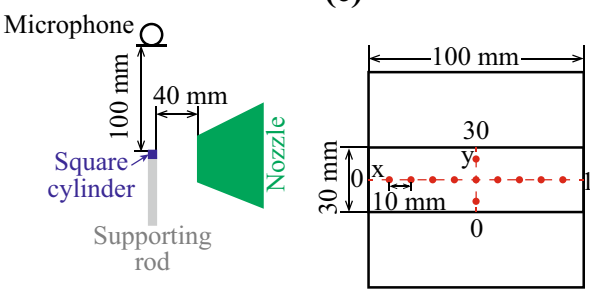

Front view

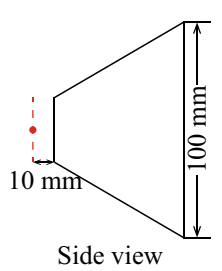

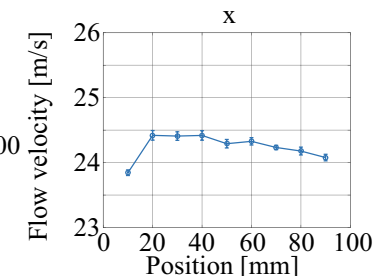

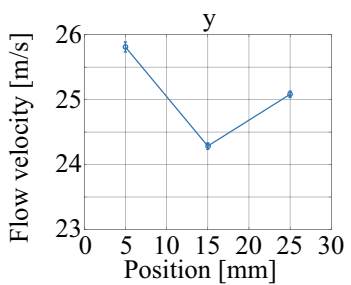


(a)

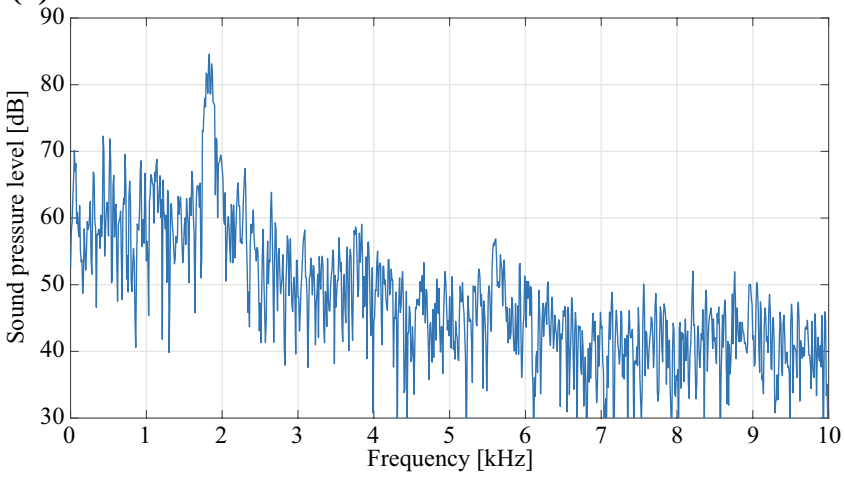

Spectra of sound captured by microphone

(b)

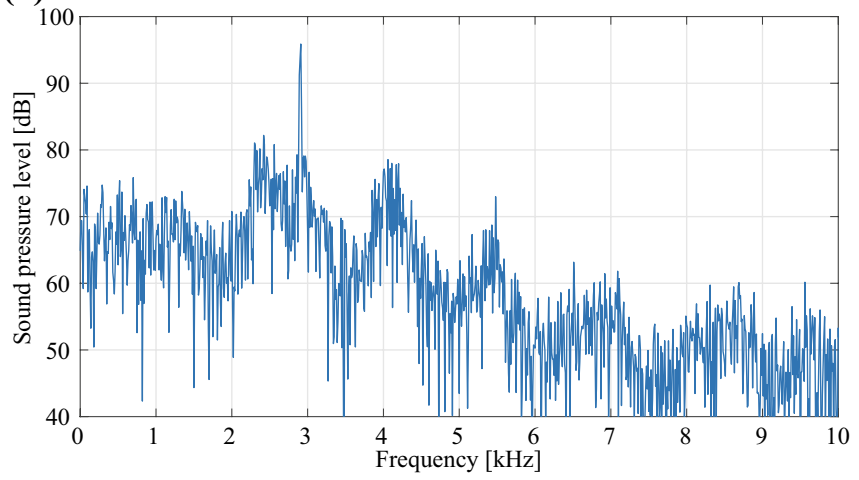

Fig. 3 Spectra of sound captured by microphone with $\mathbf{a}$ a square cylinder and $\mathbf{b}$ flat plates. The vertical axes represent sound pressure level which was calculated relative to $20 \mu \mathrm{Pa}$

the square cylinder was a tonal sound with a peak of 84.6 $\mathrm{dB}$ at $1830 \mathrm{~Hz}$ (see Fig. 3a). The Strouhal number was 0.15 . The sound radiated from the flow around the flat plates was a tonal sound with a peak of $95.9 \mathrm{~dB}$ at 2910 $\mathrm{Hz}$ (see Fig. 3b). The Strouhal number was 0.24.

\section{Visualization results}

To explain the captured PPSI data and the positional relationship of the objects and the measurement area, instantaneous images of the light intensity and phase are shown in Fig. 4. The intensity image is the average of four
Fig. 4 Images of the instantaneous light intensity and phase of light captured by PPSI for a a square cylinder and $\mathbf{b}$ flat plates
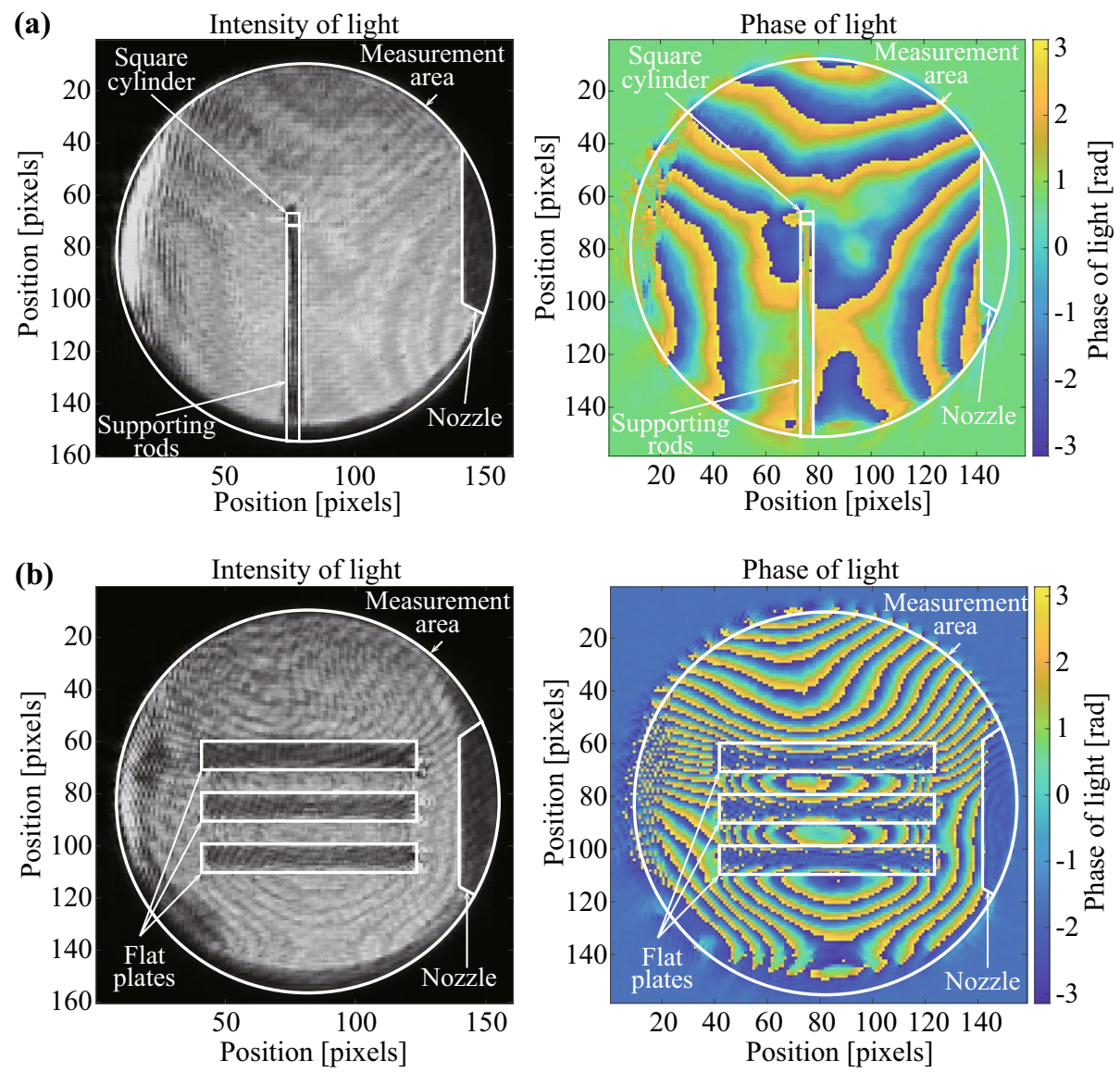
phase-shifted interference fringes captured by PPSI. The phase image was calculated from four phase-shifted interference fringes by using the hyper ellipse fitting in subspace (HEFS) method (Yatabe et al. 2017) to calculate the phase of light and reduce the noise. We can see the positions of the square cylinder and flat plates from the intensity images. The circular area is the measurement area. The shadow on the right edge is the nozzle outlet. In the light intensity image in Fig. $4 \mathrm{a}$, the vertical shadow at the center is due to the supporting rods and the square cylinder. In the light intensity image in Fig. 4b, the horizontal shadows at the center of the measurement area are the three flat plates. The light phase contains information about the sound pressure, as described in Sec. 2.1. However, the wavy pattern in the phase image was not due to the sound but rather due to the inclination and distortion of the optical flat reflecting the light at the end of the test section. We cannot directly recognize the sound in the phase image of Fig. 4 because the changes in the light phase caused by the sound were very small at approximately $\pm 0.02 \mathrm{rad}$ out of $\pm \pi \mathrm{rad}$. To visualize the sound and eliminate the effects of the optical flat inclination and distortion, band-pass filters for wrapped phases (Yatabe et al. 2018) were applied to the light phase data.

The visualized pressure distributions and waveforms captured by the microphone are shown in Figs. 5 and 6 .
Here, the pressure distributions indicate the pressure differences from the atmospheric pressures, and the pressures were summed along the optical path through the test section. The visualized pressure distribution generated by the flow around a square cylinder is shown in Figs. 5a and 6a, and also in the animation in Online Resource 1. These images were calculated from the light phase after applying a band-pass filter with a center frequency of $1830 \mathrm{~Hz}$ and a bandwidth of $200 \mathrm{~Hz}$. The center frequency was determined by the sound spectrum captured by the microphone. The dipole-like pressure distribution centered around the square cylinder can be seen in the visualized images (see Fig. 5a). Considering that the measurement area was $100 \mathrm{~mm}$ in diameter, sound waves of approximately half the wavelength were observed inside the area. In addition, flow components located on the downstream side of the square cylinder can also be visualized. The distance between negative-negative pressures of the flow components calculated from the pixel interval between the nozzle outlet and the square cylinder was approximately 11.2 $\mathrm{mm}$. The flow components are pressure fluctuations due to the $1830 \mathrm{~Hz}$ periodic vortex sheddings. The upstream flow components are pressure changes occurring at the nozzle edge. Moreover, the sound amplitude varied with time (see Fig. 6a).
Fig. 5 Frame-by-frame images of sound generated by flow around $\mathbf{a}$ a square cylinder and b flat plates. The frame rate of 20, 000 frames per second corresponds to a time step of $0.05 \mathrm{~ms}$ (a)
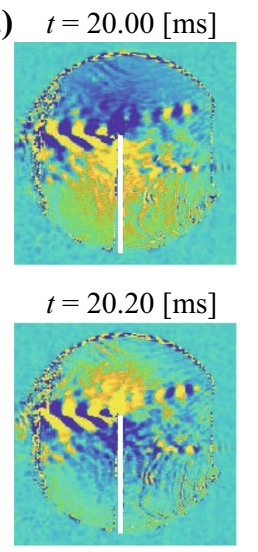

(b)
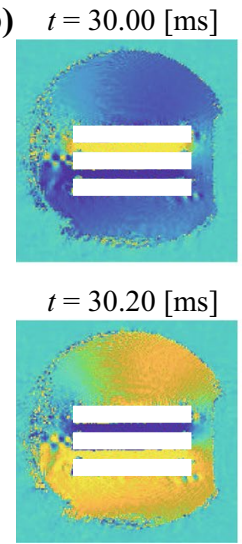
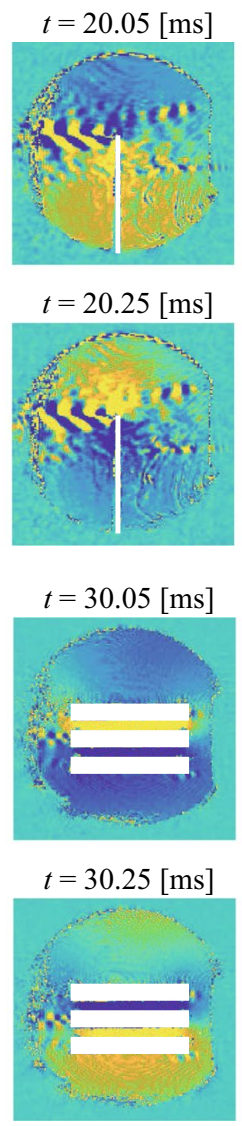
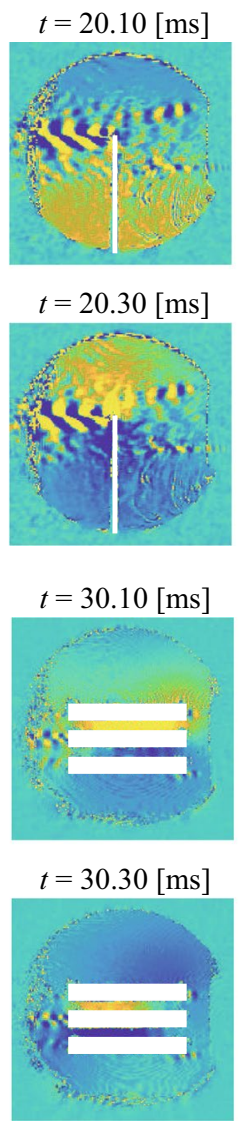
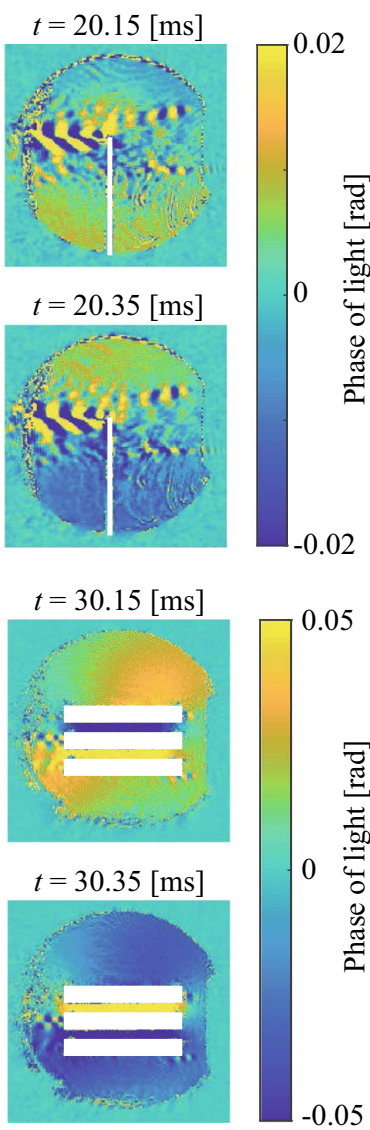

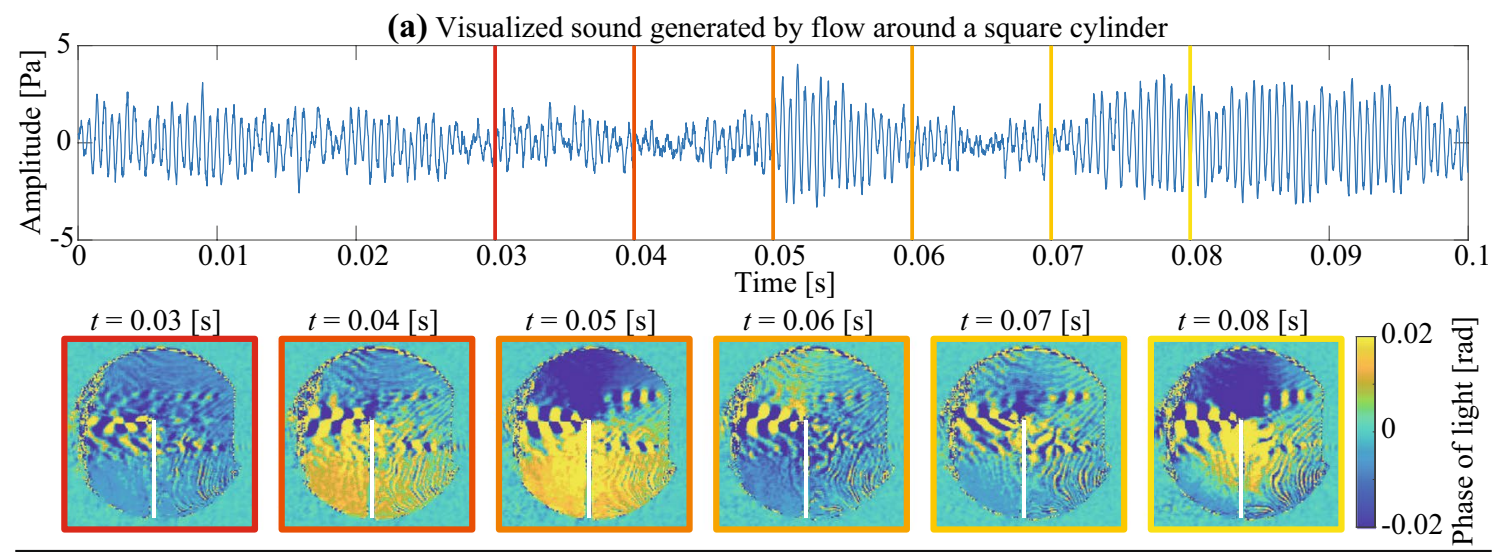

(b) Visualized sound generated by flow around flat plates
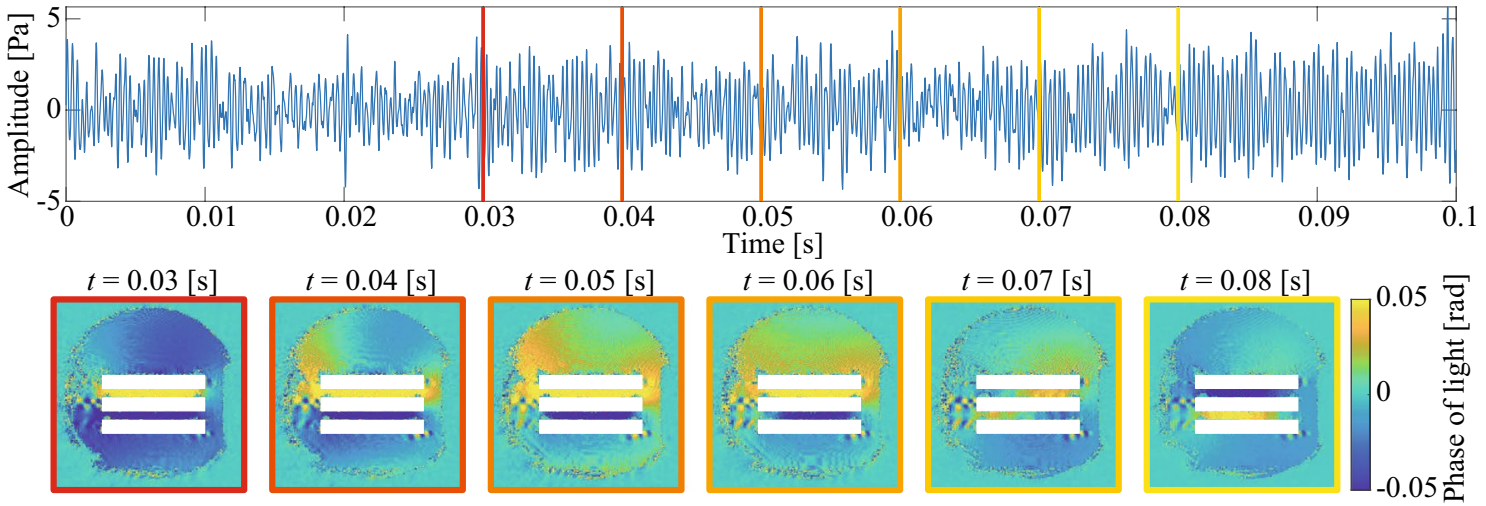

Fig. 6 Microphone signal and visualized images of sound generated by flow around a a square cylinder and $\mathbf{b}$ flat plates

The visualized pressure distribution generated by the flow around the flat plates is shown in Figs. $5 \mathrm{~b}$ and $6 \mathrm{~b}$, and also in the animation in Online Resource 2. These images were calculated from the light phase after applying a band-pass filter with a center frequency of $2910 \mathrm{~Hz}$ and a bandwidth of $50 \mathrm{~Hz}$. The center frequency was determined by the sound spectrum captured by the microphone. A narrow bandwidth was used because the $3000 \mathrm{~Hz}$ fan noise was present in the high-speed camera. The pressure distribution around and between the flat plates was visualized. When the pressure distribution between the top and middle plates was positive, the pressure distribution between the middle and bottom plates was negative and vice-verse. This should be the half-wavelength resonance in the streamwise direction because the frequency of the sound corresponds to the width of the plate $(50 \mathrm{~mm}$ without the end correction). Note that the pressure distribution along the depth direction was integrated as described in Sect. 2. Therefore, pressure fluctuation in the depth direction cannot be visualized correctly. To alleviate such situation, we set the length of the plates in the depth direction to 140 $\mathrm{mm}$ so that its resonant frequencies do not coincide with those in the streamwise direction. Indeed, the frequency spectrum in Fig. $3 \mathrm{~b}$ does not show noticeable peaks related to $140 \mathrm{~mm}$. Since the narrowband filter extracted the main resonant component related to $50 \mathrm{~mm}$, we believe that this visualized result correctly illustrates the resonant phenomena in the streamwise direction. We can also observe the pressure distributions due to the radiated sounds on the upper and lower sides of these plates. Note that the flat plates were not in a free field. As the nozzle was placed close to the plates $(10 \mathrm{~mm})$, the sound radiated from the plates was affected by its existence. By carefully watching the animation in Online Resource 2, it can be seen that the radiated sound was reflected by the nozzle placed on the right. In addition to the pressure distribution related to sound, the flow components downstream of the middle flat plates were clearly visualized. The distance between negative-negative pressures of the flow components calculated from the pixel interval between the nozzle outlet and the flat plates was approximately $7.5 \mathrm{~mm}$. The flow components are pressure fluctuations derived from periodic vortex shedding at $2910 \mathrm{~Hz}$. The upstream flow components might include pressure changes disturbed by the nozzle edge. Moreover, the sound amplitude varied with time (see Fig. 6b). 


\section{Analysis}

The visualized pressure fields include the sound and flow components. These elements should be correlated if the visualized sound was actually induced by the visualized flow components. In order to confirm the relation between the flow components and the sound, cross-correlation coefficients of the amplitude time variations were calculated. This allows the region of flow components important for sound generation to be visualized. First, the root mean square envelopes of the time-directional signals of each pixel were calculated. The window length was set to 40 samples, which corresponds to $2 \mathrm{~ms}$. Then, the crosscorrelation coefficients of the mean-subtracted envelopes between the reference pixel and the other pixels were calculated as shown in Fig. 7. The reference pixel is displayed as a red circle in the figure. The cross-correlation coefficients represent the similarity of the amplitude variations to the reference point. In the square cylinder experiment, the reference pixel was selected within the flow components to confirm that the sound pressure was related to the flow. In the flat plates experiment, the reference pixel was selected at a resonance to investigate the flow components that were affected by the resonance. Figure 7 a shows that the cross-correlation coefficients are high at the downstream, upper, and lower sides of the square cylinder. Figure $7 \mathrm{~b}$ shows that the cross-correlation coefficients are high between the flat plates, downstream of the middle plate, and upstream of the bottom plate. These results indicate that the sound and flow components changed their amplitudes in similar manners over time. Therefore, the highly correlated parts of the flow components are important for generating sound.

Focusing on the flow component time variations in the square cylinder experiment, Fig. 8 shows the change in the positive and negative pressure distributions patterns of the upper and lower flow components with time. The asymmetry of the pressure distributions can be derived from the vortex shedding asymmetry. Focusing on the asymmetric pattern in Fig. 8, the pressure distributions of the flow at the upper and lower sides of the square cylinder were vertically aligned at $t=0.05$ and $0.08 \mathrm{~s}$ but deformed and slanted at $t=0.03,0.04,0.06$, and $0.07 \mathrm{~s}$. In addition, the vertical alignment of the flow seems to correspond to the sound amplitude (see Online Resource 1). In order to investigate the relation between such alignment of the flow and sound amplitude, the pressure difference between the upper and lower sides of the flow was compared to the sound pressure amplitude. To evaluate the sound pressure separately
Fig. 7 Cross-correlation coefficients of the visualization images of the a square cylinder and $\mathbf{b}$ flat plates

Fig. 8 Visualization images of flow components
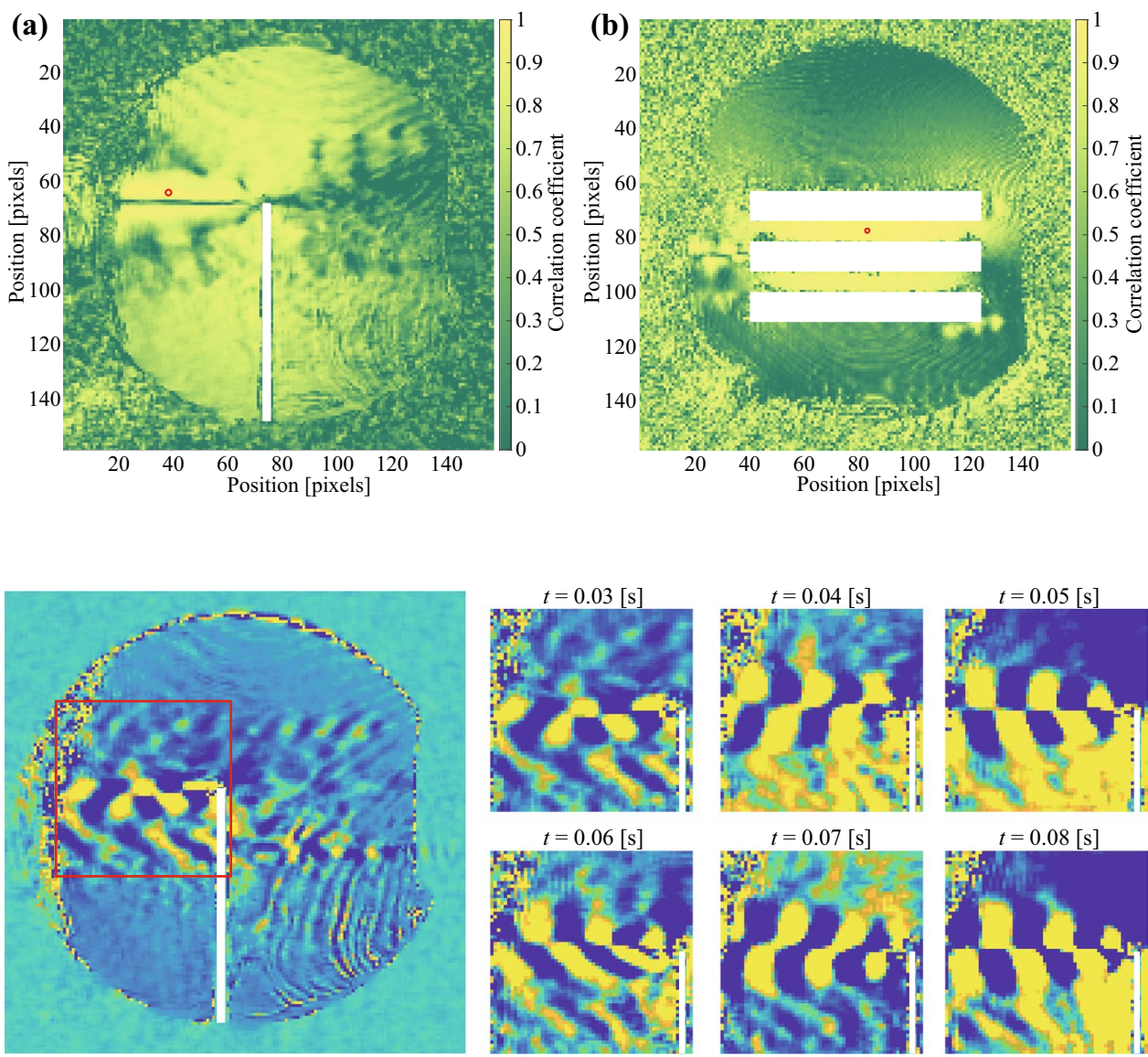

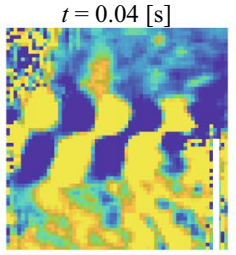

$t=0.07[\mathrm{~s}]$

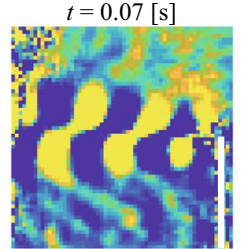

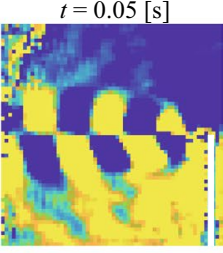

$t=0.08[\mathrm{~s}]$

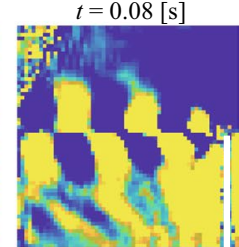


from the pressure disturbances caused by the flow, the sound pressure was extracted by spatio-temporal band-pass filtering to extract the components that satisfy the wave equation (Chitanont et al. 2017). The spatial frequencies in the sound and flow were different. Therefore, the sound pressures can be extracted using a spatio-temporal filter (Tanigawa et al. 2018, 2019). We extracted the sound pressure at $1830 \mathrm{~Hz}$ by using the spatio-temporal band-pass filter. The instantaneous images of the visualized pressure fields and the time variations of the sound amplitude and the pressure differences of the flow are shown in Fig. 9. The sound pressures at 150 pixels in the upper and lower sides of the square cylinder were selected (see blue rectangles in Fig. 9). The sound pressures were chosen from the spatio-temporal band-pass filtered images. The root mean square envelopes were calculated. The window length was set to 40 samples, which corresponds to $2 \mathrm{~ms}$. The averaged envelope is plotted as a blue line on the right side of Fig. 9. The relative amplitude of sound refers to the relative envelope values of the sound signals. The flow pressures at 150 pixels in the upper and lower sides of the square cylinder were selected (see red rectangles in Fig. 9) from the temporal band-pass filtered images, which have the same pressure distributions as Figs. 5 and 6 . To evaluate the alignment of the pressures, the signals from the upper side of the square cylinder were subtracted from the signals from the lower side. The root mean square envelopes of the subtracted pressures in the flow were calculated. The window length was set to 40 samples, which corresponds to $2 \mathrm{~ms}$. The averaged envelope is plotted as a red line on the right side of Fig. 9. The relative difference value of the flow indicates the relative envelope values of the differentiated flow pressures. The results show that the time history of the flow pressure difference was similar to that of the sound pressure amplitude. Because vertically aligned flow indicates a larger difference magnitude and slanted flow a smaller difference amplitude, the slow variations of the sound pressure should be due to the alignment of the flow pressure distributions.
Focusing on the resonant sound in the flat plates experiment, the phase difference of the sound between the upstream and downstream sides can be confirmed in the visualized images. To investigate the characteristics of the resonant sound, the Fourier phase of the sound was calculated as the complex arguments of the time-directional Fourier-transformed signals at $2910 \mathrm{~Hz}$ for each pixel. An image of the Fourier phase is shown in Fig. 10a. Focusing on the Fourier phase between the flat plates, the values slightly increased along the mainstream direction. The values inside the black rectangles were extracted. The averaged values are displayed as circles, and the standard variations are displayed as error bars in Fig. 10b. Between the upstream to the downstream, the Fourier phase decreased by 0.14 and 0.32 rad in the upper and lower slits, respectively. The Fourier phase difference between upstream and downstream might be caused by the flow. This result suggests that the Fourier phase difference of the resonant sound was swept along the flow.

\section{Conclusions}

We visualized the aerodynamic sound generated by the flow around the vicinity of a square cylinder and flat plates using PPSI. The dipole-like pressure distributions around the square cylinder and the half-wavelength resonant pressure between flat plates were visualized in the respective experiments. This also allowed the flow components important for generating the sound to be visualized. The time variations of the sound and flow amplitudes were correlated. Such a relation between the flow and sound around and/or inside the objects can be only revealed by non-intrusive visualization of pressure fields, as in the proposed method. Further investigations should be conducted to evaluate the observed phenomena such as the relation between sound amplitudes and the flow disturbances in the square cylinder experiment, and the sound Fourier phase declinations along the mean flow.
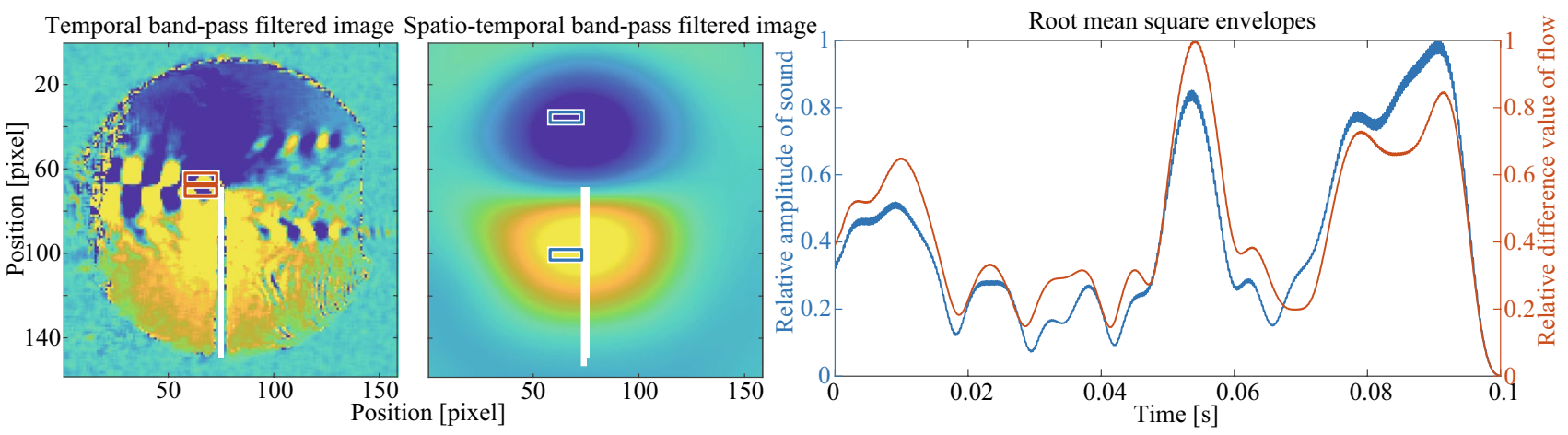

Fig. 9 Comparison of root mean square envelopes between sound and flow difference 
(a)

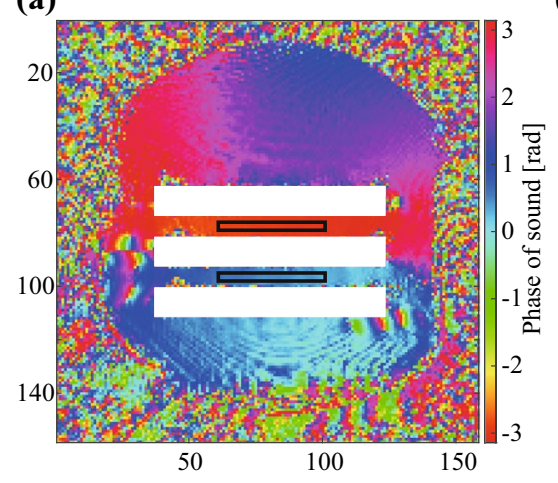

(b)

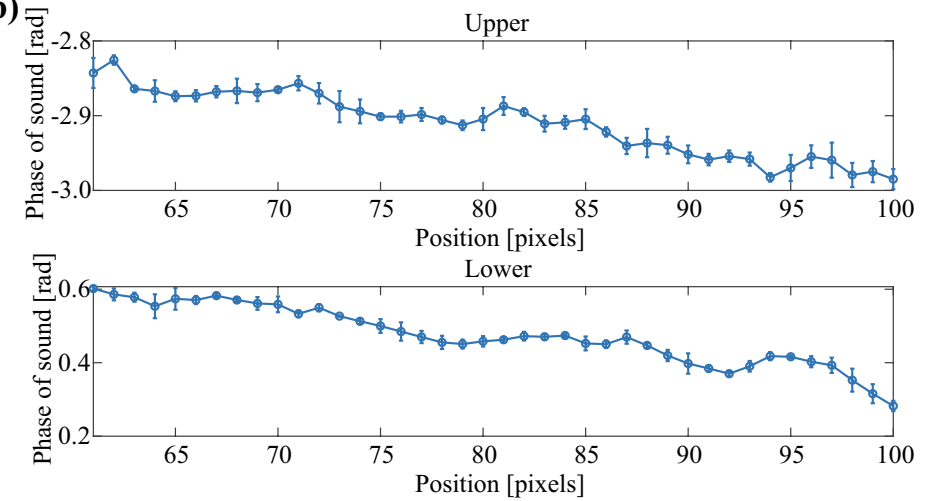

Fig. 10 Phase distribution and phase values between the flat plates at $2910 \mathrm{~Hz}$

Open Access This article is licensed under a Creative Commons Attribution 4.0 International License, which permits use, sharing, adaptation, distribution and reproduction in any medium or format, as long as you give appropriate credit to the original author(s) and the source, provide a link to the Creative Commons licence, and indicate if changes were made. The images or other third party material in this article are included in the article's Creative Commons licence, unless indicated otherwise in a credit line to the material. If material is not included in the article's Creative Commons licence and your intended use is not permitted by statutory regulation or exceeds the permitted use, you will need to obtain permission directly from the copyright holder. To view a copy of this licence, visit http://creativecommons.org/licenses/by/4.0/.

\section{References}

Adamczyk AA, Rimai L (1988) 2-Dimensional particle tracking velocimetry (PTV): technique and image processing algorithms. Exp Fluid 6(6):373-380

Adrian RJ (1991) Particle-imaging techniques for experimental fluid mechanics. Annu Rev Fluid Mech 23(1):261-304

Awatsuji Y, Sasada M, Kubota T (2004) Parallel quasi-phase-shifting digital holography. Appl Phys Lett 85(6):1069-1071

Chitanont N, Yatabe K, Ishikawa K, Oikawa Y (2017) Spatio-temporal filter bank for visualizing audible sound field by Schlieren method. Appl Acoust 115:109-120

Hargather MJ, Settles GS, Madalis MJ (2010) Schlieren imaging of loud sounds and weak shock waves in air near the limit of visibility. Shock Waves 20(1):9-17

Ishikawa K, Yatabe K, Chitanont N, Ikeda Y, Oikawa Y, Onuma T, Niwa H, Yoshii M (2016) High-speed imaging of sound using parallel phase-shifting interferometry. Opt Express 24(12):12922-12932

Knisely CW (1990) Strouhal numbers of rectangular cylinders at incidence: a review and new data. J Fluid Struct 4(4):371-393

Løkberg OJ (1994) Sound in flight: measurement of sound fields by use of TV holography. Appl Opt 33(13):2574-2584

Millerd JE, Brock NJ, Hayes JB, North-Morris MB, Novak M, Wyant JC (2004) Pixelated phase-mask dynamic interferometer. In: Creath K, Schmit J (eds) Interferometry XII: techniques and analysis, vol 5531. International Society for Optics and Photonics, SPIE, Bellingham, pp 304-314
Oikawa Y, Goto M, Ikeda Y, Takizawa T, Yamasaki Y (2005) Sound field measurements based on reconstruction from laser projections. In: Proc. IEEE int. conf. acoust. speech signal process, vol 4, pp iv/661-iv/664

Onuma T, Otani Y (2014) A development of two-dimensional birefringence distribution measurement system with a sampling rate of 1.3 MHz. Opt Commun 315:69-73

Tanigawa R, Ishikawa K, Yatabe K, Oikawa Y, Onuma T, Niwa H (2018) Optical visualization of a fluid flow via the temperature controlling method. Opt Lett 43(14):3273-3276

Tanigawa R, Yatabe K, Oikawa Y (2019) Guided-spatio-temporal filtering for extracting sound from optically measured images containing occluding objects. In: Proc. IEEE int. conf. acoust. speech signal process, pp 945-949

Torras-Rosell A, Barrera-Figueroa S, Jacobsen F (2012) Sound field reconstruction using acousto-optic tomography. J Acoust Soc Am 131(5):3786-3793

van den Berg GP (2006) Wind-induced noise in a screened microphone. J Acous Soc Am 119(2):824-833

Yatabe K, Ishikawa K, Oikawa Y (2016) Signal processing for optical sound field measurement and visualization. Proc Meet Acoust 29(1):020010

Yatabe K, Ishikawa K, Oikawa Y (2017) Simple, flexible, and accurate phase retrieval method for generalized phase-shifting interferometry. J Opt Soc Am A 34(1):87-96

Yatabe K, Tanigawa R, Ishikawa K, Oikawa Y (2018) Time-directional filtering of wrapped phase for observing transient phenomena with parallel phase-shifting interferometry. Opt Express 26(11):13705-13720

Yokoyama H, Iida A (2017) Direct and hybrid aeroacoustic simulations around a rectangular cylinder, chap 18. IntechOpen, London, pp $327-348$

Yokoyama H, Kitamiya K, Iida A (2013) Flows around a cascade of flat plates with acoustic resonance. Phys Fluids 25(10):106104

Publisher's Note Springer Nature remains neutral with regard to jurisdictional claims in published maps and institutional affiliations. 


\section{Affiliations}

\section{Risako Tanigawa $^{1} \cdot$ Kohei Yatabe $^{1} \cdot$ Yasuhiro Oikawa $^{1}$}

Kohei Yatabe

k.yatabe@asagi.waseda.jp

Yasuhiro Oikawa

yoikawa@waseda.jp
Department of Intermedia Art and Science, Waseda University, 3-4-1 Ohkubo, Shinjuku-ku, Tokyo 169-8555, Japan 